International Journal of Integrative Pediatrics and Environmental Medicine, V7, 2022

\title{
Original Review
}

\section{PHYSICAL INACTIVITY IN YOUTH DURING COVID-19: A NARRATIVE REVIEW}

\author{
Tiffany Field, $\mathrm{PhD}^{1}{ }^{*}$
}

\begin{abstract}
Author information: 1. University of Miami/Miller School of Medicine and Fielding Graduate University.
\end{abstract}
Received: 11-21-2021; Accepted: 12-22-2021; Published: 01-12-2022

\begin{abstract}
The prevalence of physical inactivity in youth during COVID-19 has varied across countries and depending on age of the youth and whether the data were collected during lockdown versus nonlockdown. In studies that compared activity pre- and during COVID, activity decreased in $40-59 \%$ of youth. Several negative effects have been noted including excessive screen time, sleep problems, over-eating, and affective disturbances. Physical inactivity was a significant correlate of all of these problems. Relatively little intervention research has been conducted and underlying mechanisms have not been explored. As in most of the COVID-19 literature, this research has the limitations of being typically crosssectional and self-reported.
\end{abstract}

Keywords Physical inactivity, youth, COVID-19, screen time, sleep problems, depression

INTRODUCTION Research on physical activity in youth during COVID-19 is relatively sparse compared to the same literature on adults. In the literature search for this narrative review several terms were entered including physical activity, inactivity, exercise, and sedentary behavior in youth during COVID-19. This search yielded 52 papers, but when exclusion criteria were considered including qualitative and case studies as well as papers in non-English, only 26 papers were included. The literature can be divided among the six areas of prevalence, effects or correlates, predictors or risk factors, intervention studies, and underlying mechanisms.

PREVALENCE OF PHYSICAL INACTIVITY The prevalence of physical inactivity among youth has varied by country, by lockdown versus non-lockdown, and by age. It has been referred to in the literature as physical activity, inactivity, and sedentary behavior. In a study from Ireland, physical activity decreased in $50 \%$ of 12 to 18 -year-olds ( $N=1214$ ) during a lockdown [1]. Physical activity increased in 20\%, and $30 \%$ showed no change. Those who showed a decrease in physical activity were more frequently

\footnotetext{
*Corresponding author: Tiffany Field, 2889 McFarlane Rd, Miami, FL 33133, 305-975-5029.
}

Email address: tfield @med.miami.edu overweight or obese. Less physical activity was reported by those who also said they had less time and their workout club was closed. Greater activity was noted in those who said they had more time and were not in school. The reasons for less and more physical activity were both related to time.

In a study from Pakistan ( $\mathrm{N}=6349$ to 13-year-old youth), sufficient activity decreased from $69 \%$ before the pandemic to only $29 \%$ during the pandemic [2]. In this study, the West British children were more active than those of Pakistani heritage or other ethnicities (34\% versus $23 \%$ versus $23 \%$ ). Those who were more active left home more frequently and for longer periods of time. Being sufficiently active was related to the frequency, duration, and type of activity as well as the distance from home where the activity took place.

In a study from Gungzhou, China on primary, secondary, and high school youth ( $N=10,933$, median age equals 13 years), 50\% engaged in less than 15 minutes of physical activity per day in light, moderate, or vigorous exercise and $59 \%$ had a decrease in physical activity during the pandemic compared with before the pandemic [3]. Several correlates were noted including that $80 \%$ showed an increase in screen time and $81 \%$ of the students spent more than five hours on screen time per day. They also reported that sleep problems were experienced by $39 \%$ of 
International Journal of Integrative Pediatrics and Environmental Medicine, V7, 2022

the sample. In a study from Switzerland on university students, $37 \%$ were not engaging in physical activity [4]. As another measure of activity, $36 \%$ were reportedly sitting more than eight hours per day, and $8 \%$ of the university students did not adhere to alcohol consumption recommendations. They also reported that sleep quality was not good in $45 \%$ of the students even though the median sleep time was eight hours per day.

\section{EFFECTS/CORRELATES OF PHYSICAL INACTIVITY Several} effects or correlates have been noted for physical inactivity during COVID-19 including excessive sedentary time, increased screen time, and changes in sleep time, overeating, and overweight effects. In addition, significant changes in mood occurred, including increased anxiety, depression and negative mood. These are probably most accurately referred to as correlates given that direction of effects cannot be determined from these predominantly cross-sectional data. Physical inactivity and these other behaviors are likely to be reciprocal or bi-directional.

SEDENTARY BEHAVIOR Sedentary behavior may be considered synonymous with physical inactivity. In a study on physical activity in adolescents from 49 middle schools in China ( $\mathrm{N}=4898)$, moderate to vigorous physical activity occurred on average 23 minutes per day while sedentary behavior occurred 363 minutes per day, more than 15.7 times the time spent on physical activity [5]. In a mixed linear regression, less physical activity was related to greater mood disturbance. In another study from China ( $N=10,000$ youth), physical inactivity was related to increased sedentary behavior, screen time, and sleep time [6]. The reduction in moderate/vigorous intensity physical activity was also related to a decrease in leisure time and leisure-time walking. Similar data were reported in another study from China ( $N=10,082$ high school and university students), suggesting that decreased activity was related to increased sedentary time, screen time, and sleep time [7].

INCREASED SCREEN TIME Increased screen time is another obvious correlate of decreased physical activity in youth during COVID-19. In a study from Italy $(\mathrm{N}=41)$, the decrease in time spent in activity averaged 2.3 hours per week while the screen time increased 4.85 hours per day, suggesting that the increase in screen time was greater than the reduction in physical activity [8]. These changes were also correlated with an increase in sleep time by 0.65 hours per day and an increase in unhealthy diet that included more snacks, red meat, and sugar drinks. Similar findings have been reported in other countries including a United States' study from California in which children ( $N=649$ to 15-year-olds) who engaged in less activity also engaged in more screen time and experienced more anxiety [9]. These data were derived from 24-hour physical activity recalls, and the State Anxiety Inventory for Children [10]. Children who engaged in greater physical activity also engaged in less screen time and experienced less anxiety. In a study from China ( $\mathrm{N}=1687$ among 12th graders), physical activity of 150 minutes duration each week was related to less screen time and less negative mood, as well as fewer conflicts with parents [11]. University students in Lithuania $(\mathrm{N}=230)$ also showed decreased activity during COVID-19 and increased Internet browsing and sleep duration [12].

Similar correlations were noted in a study from Germany ( $N=1711,4$ to 17-year-olds) during a lockdown [13]. In this case, the increase in screen time accompanied a decrease in sports activity. And in a study from Pakistan ( $N=225$ high school and college adolescents) during lockdown, screen time was significantly negatively correlated with physical activity $(-0.34)$ [14]. In a study from Greece $(\mathrm{N}=397)$ on children and parents across 63 municipalities, reduced physical activity was associated with both increased screen time and increased sleep duration [15].

\section{INCREASED SLEEP TIME BUT DECREASED SLEEP QUALITY}

Decreased sleep quality has also been related to a reduction in physical activity. These relationships were reported for youth in China [6,7], Germany [13], Pakistan [14], and Italy [8]. In a study from Poland on 6 to 15-yearolds $(\mathrm{N}=1016$,$) a comparison between physical activity$ from before the pandemic to during a lockdown showed a decrease in sleep quality associated with a decrease in physical activity [16]. In Switzerland, at least $45 \%$ of university students reported poor sleep quality, although the median sleep time was eight hours [4]. Decreased sleep quality is the most frequently noted correlate of reduced physical activity [4].

Although sleep quality frequently decreased with reduced physical activity, sleep duration increased with reduced physical activity in the same studies. Negative correlations have been reported in the studies from China $[6,7]$ and Pakistan [14], although the negative correlation was weak in the Pakistan study $(r=-0.16)$. The study on university students from Lithuania also showed a relationship 
International Journal of Integrative Pediatrics and Environmental Medicine, V7, 2022

between reduced physical activity and increased sleep duration [12]. In still another study on university students, a decrease in sleep duration occurred as physical activity decreased [17]. This combination in students from the US led to an increase in stress.

DIETARY CHANGES, WEIGHT GAIN, OVERWEIGHT, AND OBESITY Dietary changes related to reduced physical activity as well as weight gain, overweight, obesity or most typically a combination of these. In a sample of Europeans and Latin Americans ( $\mathrm{N}=726$ 16-19-year-olds), for example, decreased physical activity was related to greater consumption of processed food during the pandemic [18]. These data were based on the International Physical Activity Questionnaire [19]. In the study from Italy already described, reduced physical activity was associated with an unhealthy diet including red meat, potato chips, and sugar drinks [8]. In contrast, in another study from Italy, exercise was related to a healthy diet of fruits, vegetables, and fish [20]. Fish consumption was significantly related to physical activity that occurred four times per week and a reduction in bodyweight in a study on children from Chile [21]. In that sample, body weight was correlated with consumption of fried foods and alcohol consumption was negatively related to physical activity.

It is not surprising that across the pandemic, mean body mass index (BMI) increased [6]. For example, in a study from China, the researchers reported that mean BMI increased for everyone, and significant increases were noted for the prevalence of overweight (21-25\%) and obesity (11-13\%) [6].

In a study of 827 German university students, $27 \%$ gained weight but, surprisingly, $22 \%$ lost weight [22]. In this study, consumption of bakery products, pasta, meat, and snacks notably increased. Less frequent cooking with fresh ingredients, more smoking, and more alcohol consumption were significant predictors of less physical activity. Surprisingly, in the study from Greece, an increase was noted in healthy food consumption and a decrease in unhealthy food consumption, although there was an increase in body weight in $35 \%$ of the children [15]. In a regression analysis, increased body weight was explained by the decreased physical activity and increased consumption of breakfast foods and snacks.
NEGATIVE MOOD STATES Negative mood states have been associated with decreased physical activity, with excessive screen time and eating problems. For example, in China, negative moods were associated with physical activity of $\mathbf{1 5 0}$ minutes duration per week which, in turn, was related to increased screen time [11]. And, in research from Italy, exercise mediated the negative relationship between mood states and healthy diet (fruits, vegetables, and fish) [20]. Negative mood states as measured by the Profile of Mood States [23] have been associated with sedentary activity [5]. In the study from Pakistan, as many as $64 \%$ of high school and college age adolescents reputedly had "psychological disease" [14]. Psychological disease was negatively correlated with physical activity $(r=0-.34)$ but also positively correlated with screen time and distress. And "mental health" has been positively correlated with physical activity in a review of studies on physical activity as it relates to mental health during COVID-19 [24].

In studies that focused on physical activity and anxiety, more physical activity was related to less anxiety as well as less sedentary time and leisure screen time [9]. In this study physical activity was measured as 24 -hour recall and anxiety was measured by the State Anxiety Inventory for Children in 9 to 15-year-olds [9]. In a study from the US using the GAD-7 (Generalized Anxiety and Depression-7 items) [25], and the PHQ-9 (Physical Health Questionnaire9 items) [26] for depression, both anxiety and depression were related to scores on the Pediatric Functional Activity Brief Scale $[27,28]$. Depression was correlated with less exercise in this study on US adolescent athletes, where $74 \%$ of those who engaged in team sports experienced depression while $65 \%$ engaged in individual sports were depressed [28]. Interestingly, engaging in exercise with others outside was a significant buffer for depression in a Facebook survey during COVID-19 [29]. Thus, missing exercise with others has seemed to be a greater stressor and exercising with others has been a greater buffer during COVID-19.

PREDICTORS/RISK FACTORS FOR PHYSICAL INACTIVITY Just as effects of inactivity have been arbitrarily labeled effects when they are probably correlates, predictors or risk factors could also be called correlates of inactivity. Just as negative mood states including depression have been identified as effects of inactivity, they may also be 
International Journal of Integrative Pediatrics and Environmental Medicine, V7, 2022

correlates or causal factors. Some surprising demographics have also been considered risk factors, including neighborhood density and maternal education.

NEIGHBORHOOD DENSITY In at least two studies on physical activity of youth during COVID-19, density of the neighborhood was a predictor for physical activity level. In a study from Germany ( $\mathrm{N}=1711$, 4-17-year-olds), increased physical activity during the first lockdown was greater in more densely populated areas [30]. In another physical activity study, greater activity was noted outside in low density neighborhoods, but also in high density neighborhoods with parks in Canada [31].

MATERNAL EDUCATION Another surprising predictor variable was mothers' education. In a study on European and Latin American adolescents ( $N=720,16$ to 19-yearolds), those whose mothers had more education were less active, especially in Latin America [18]. This finding is difficult to interpret, although it may relate to more family time being spent on the Internet and less time engaging in physical activity, consistent with several studies already described on the relationship between excessive screen time and reduced physical activity [32].

NEGATIVE MOOD STATES Negative mood states including depression have also been considered predictors of physical inactivity. In a study from the US, depression and physical inactivity were occurring more often for those engaging in team sports than individual sports, likely because team sports were not occurring during lockdowns [28]. In the study from Italy, exercise mediated the relationship between mood states and healthy diets [20]. Surprisingly, this is the only mediation model study that appeared on relationships between physical inactivity or exercise and other variables in this literature search on youth during COVID-19, even though mediation/ moderation model data analyses have become a popular form of analysis.

In a study of children and youth from Canada ( $N=1472,5$ 11 and 12-17-year-old youth who met 24-hour movement guidelines), several predictor variables were identified, highlighting the inter-relationships of physical activity with many factors [33]. Based on decision tree modeling to generate physical activity profiles, the predictors were increased screen time, income, increased outdoor sports, male gender, parents who were younger than 43-yearsold, and increased sleep duration.
INTERVENTION FOR PHYSICAL INACTIVITY DURING COVID-19 Only two intervention studies could be found in the COVID-19 literature on physical activity in youth. This is not surprising because most of the literature derives from early in the pandemic and interventions typically require more time to develop. In a study from China, a peer-to-peer live-streaming intervention was provided for grade 7 students from 12 middle schools $(\mathrm{N}=896)$ [34]. The intervention group received health information that promoted exercise and the control group received health information without the exercise promotion [34]. In addition to a decrease in anxiety, a decrease in eyestrain occurred. The decreased eyestrain likely related to the students spending more time exercising than straining their eyes at their computers or on their cell phones.

In another intervention study called the "Walking in School Study", 12-to-14-year-old Irish students ( $\mathrm{N}=281)$ were engaged in extra walking [35]. Surprisingly, there was no change in physical activity, social media use, or sleep quality, but motivation for exercise and health-related quality of life increased.

POTENTIAL UNDERLYING MECHANISMS FOR PHYSICAL INACTIVITY EFFECTS Some potential underlying effects of physical activity/exercise have been suggested elsewhere [36]. In research comparing massage therapy and yoga, for example, similar effects were noted, suggesting that moving the skin increases vagal activity which in turn slows the nervous system (e.g., decreases heart rate and increases electroencephalograph theta waves associated with relaxation) [36]. The slowing of the nervous system is associated with reductions in anxiety, depression, and stress hormones (e.g. cortisol). The reduction in cortisol in turn results in enhanced immune function (e.g. increased natural killer cell number and natural killer cell activity that kill bacterial and viral cells). In addition, exercise has been found to increase serotonin, the body's natural antidepressant and anti-pain neurotransmitter [36]. Future studies that measure the hormonal and immune effects of pandemic physical activity/exercise could inform prevention/intervention research.

METHODOLOGICAL LIMITATIONS Several methodological limitations can be noted for this literature, not unlike much of the other COVID-19 research that was planned and executed in a rapid fashion as the lockdowns were occurring. First, there is significant variability across the various cultures in terms of virtually every parameter of 
International Journal of Integrative Pediatrics and Environmental Medicine, V7, 2022

the studies including time of the pandemic, lockdown versus non-lockdown, age of the participants, the measures and the data analyses. This variability is likely the reason that meta-analyses have not yet appeared in the literature on physical inactivity in youth during COVID-19. Variability is also characteristic of the baseline measures which barely exist because the start dates of the lockdowns were not anticipated by the researchers. These include baseline activity levels and their many covariates, for example, baseline weight, overweight, obesity, screen time, and mood states. Given that the timing of the pandemic and lockdowns was unpredictable, collecting baseline or longitudinal data was difficult or not possible, especially for anonymous surveys that constitute the bulk of this literature. For those reasons, very few studies are longitudinal, especially on measures that were collected pre-pandemic and during the pandemic.

Furthermore, the surveys were subject to various types of biases including recall accuracy, social desirability ratings, and reporting by parents rather than the youth participants who may be less familiar with activity levels than the youth were themselves. Physical activity scales and mean daily activity levels have been used more frequently than 24-hour activity recordings and the World Health Organization standard for activity was rarely assessed or mentioned.

In addition, several confounding variables for activity level may not be correctable by their inclusion as covariates, including screen time, diet/eating habits, and mood states, although they have been considered risk factors and effects of physical inactivity and were labeled correlates in this narrative review. Researchers frequently focused on similar variables but measured them in different ways as from a negative or a positive valence. For example, activity was measured as positive as in hours of exercise or activity or negative as in being inactive or sedentary and diet as healthy versus unhealthy foods. And the predictor/risk variables are just as likely effects variables or vice versa given that many of the variables are reciprocal or bidirectional.

Despite these methodological limitations, this literature highlights the prevalence of physical inactivity and the correlates of physical inactivity in youth during COVID-19. It reveals the need for research on exercise intervention programs during pandemics like COVID-19.

\section{REFERENCES}

1. Ng K, Cooper J, McHale F, Clifford J, Woods C. Barriers and facilitators to changes in adolescent physical activity during COVID-19. BMJ Open Sport Exerc Med 2020;5:6:e000919. doi: 10.1136/bmjsem-2020000919.

2. Bingham DD, Daly-Smith A, Hall J, et al. Covid-19 lockdown: Ethnic differences in children's selfreported physical activity and the importance of leaving the home environment; a longitudinal and cross-sectional study from the Born in Bradford birth cohort study. Int J Behav Nutr Phys Act 2021;18:117. doi: 10.1186/s12966-021-01183-y.

3. Guo YF, Liao MQ, Cai WL, et al. Physical activity, screen exposure and sleep among students during the pandemic of COVID-19. Sci Rep. 2021 Apr 20;11:8529. doi: 10.1038/s41598-021-88071-4.

4. Taeymans J, Luijckx E, Rogan S, Haas K, Baur H. Physical activity, nutritional habits, and sleeping behavior in students and employees of a Swiss university during the COVID-19 lockdown period: Questionnaire survey study. JMIR Public Health Surveill 2021;13;7(4):e26330. doi: 10.2196/26330.

5. Kang S, Sun Y, Zhang X, Sun F, Wang B, Zhu W. Is physical activity associated with mental health among Chinese adolescents during isolation in COVID-19 pandemic? J Epidemiol Glob Health 2021;11:26-33. doi: 10.2991/jegh.k.200908.001.

6. Yang S, Guo B, Ao L, et al. Obesity and activity patterns before and during COVID-19 lockdown among youths in China. Clin Obes 2020;10:e12416. doi:

10.1111/cob.12416.

7. Jia $P$, Zhang L, Yu W, et al. Impact of COVID-19 lockdown on activity patterns and weight status among youths in China: the COVID-19 Impact on Lifestyle Change Survey (COINLICS). Int J Obes (Lond) 2021;45:695-9. doi: 10.1038/s41366-020-00710-4.

8. Pietrobelli A, Pecoraro L, Ferruzzi A, et al. Effects of COVID-19 ILockdown on lifestyle behaviors in children with obesity living in Verona, Italy: A longitudinal study. Obesity (Silver Spring) 2020;28:1382-5. doi: 10.1002/oby. 22861.

9. Alves JM, Yunker AG, DeFendis A, Xiang AH, Page KA. Associations between affect, physical activity, and anxiety among US children during COVID-19. Pediatr 
International Journal of Integrative Pediatrics and Environmental Medicine, V7, 2022

Obes 2021;15:e12786 doi:

10.1101/2020.10.20.20216424.

10. Spielberger CD, Gorsuch RL, Lushene R, et al. The preliminary test manual for the State-Trait Anxiety Inventory for Children. Menlo Park (CA): Mind Garden, Inc; 1983.

11.Xiao S, Yan Z, Zhao L. Physical activity, screen time, and mood disturbance among Chinese adolescents during COVID-19. J Psychosoc Nurs Ment Health Serv 2021;59:14-20. doi: 10.3928/02793695-20201104-04.

12. Baceviciene M, Jankauskiene R. Changes in sociocultural attitudes towards appearance, body image, eating attitudes and behaviours, physical activity, and quality of life in students before and during COVID-19 lockdown. Appetite 2021;166:105452. doi: 10.1016/j.appet.2021.105452.

13. Schmidt SCE, Anedda B, Burchartz A, et al. Physical activity and screen time of children and adolescents before and during the COVID-19 lockdown in Germany: a natural experiment. Sci Rep 2020;10:21780. doi: 10.1038/s41598-020-78438-4.

14. Awais MA, Chaudhery MM, Khan MS, et al. Factors contributing to distress among school and collegegoing adolescents during COVID-19 lockdown: A crosssectional study conducted in Sibi Balochistan, Pakistan. J Educ Health Promot 2021;10:317. doi: 10.4103/jehp.jehp_1313_20.

15. Androutsos O, Perperidi M, Georgiou C, Chouliaras G. Lifestyle changes and determinants of children's and adolescents' body weight increase during the first COVID-19 lockdown in Greece: The COV-EAT Study. Nutrients 2021;13:930. doi: 10.3390/nu13030930.

16.Łuszczki E, Bartosiewicz A, Pezdan-Śliż l, et al. Children's eating habits, physical activity, sleep, and media usage before and during COVID-19 pandemic in Poland. Nutrients 2021;13:2447. doi:

10.3390/nu13072447.

17. Moriarty T, Bourbeau K, Fontana F, McNamara S, Pereira da Silva $M$. The relationship between psychological stress and healthy lifestyle behaviors during COVID-19 among students in a US Midwest university. Int J Environ Res Public Health 2021;18:4752. doi: 10.3390/ijerph18094752.

18. Ruíz-Roso MB, de Carvalho Padilha P, MatillaEscalante DC, et al. Changes of physical activity and ultra-processed food consumption in adolescents from different countries during Covid-19 pandemic: An observational study. Nutrients 2020;12:2289. doi: 10.3390/nu12082289.

19. Craig $C L$, Marshall $A L$, Sjöström $M$, et al. International physical activity questionnaire: 12-country reliability and validity. Med Sci Sports Exerc. 2003;35:1381-95. doi: 10.1249/01.MSS.0000078924.61453.FB.

20.Amatori S, Donati Zeppa S, Preti A, et al. Dietary habits and psychological states during COVID-19 home isolation in Italian college students: The role of physical exercise. Nutrients 2020;12:3660. doi: 10.3390/nu12123660.

21. Reyes-Olavarría D, Latorre-Román PÁ, GuzmánGuzmán IP, Jerez-Mayorga D, Caamaño-Navarrete F, Delgado-Floody P. Positive and negative changes in food habits, physical activity patterns, and weight status during COVID-19 confinement: Associated factors in the Chilean population. Int J Environ Res Public Health 2020;17:5431. doi: 10.3390/ijerph17155431.

22. Palmer K, Bschaden A, Stroebele-Benschop N. Changes in lifestyle, diet, and body weight during the first COVID 19 'lockdown' in a student sample. Appetite 2021;167:105638. doi: 10.1016/j.appet.2021.105638.

23. McNair D, Lorr M, Droppleman L. Manual for the Profile of Mood States. San Diego: Educational and Industrial Testing Service; 1971. P. 27.

24. Okuyama J, Seto S, Fukuda Y, et al. Mental health and physical activity among children and adolescents during the COVID-19 pandemic. Tohoku J Exp Med 2021;53:203-15. doi: 10.1620/tjem.253.203.

25. Kertz S, Bigda-Peyton J, Bjorgvinsson T. Validity of the Generalized Anxiety Disorder-7 scale in an acute psychiatric sample. Clin Psychol Psychother. 2013;20:456-64. doi: 10.1002/cpp.1802.

26. Kroenke K, Spitzer RL, Williams JB. The PHQ-9: validity of a brief depression severity measure. J Gen Intern Med. 2001; 16:606-13.

27. Fabricant PD, Robles A, Downey-Zayas T, et al. Development and validation of a pediatric sports activity rating scale: the Hospital for Special Surgery Pediatric Functional Activity Brief Scale (HSS PediFABS). Am J Sports Med. 2013;41:2421-9. doi: $10.1177 / 0363546513496548$. 
International Journal of Integrative Pediatrics and Environmental Medicine, V7, 2022

28. McGuine TA, Biese KM, Petrovska L, et al. Mental health, physical activity, and quality of life of US adolescent athletes during COVID-19-related school closures and sport cancellations: A study of 13000 athletes. J Athl Train 2021;56:11-9. doi: 10.4085/10626050-0478.20.

29 Field T. Anxiety and depression in youth during COVID19: A narrative review. J Psychiatry Research Reviews and Reports. 2021;3:1-8. doi.org/10.47363/JPSRR/2021(3)126.

30. Nigg C, Oriwol D, Wunsch K, et al. Population density predicts youth's physical activity changes during Covid-19 - Results from the MoMo study. Health Place 2021;70:102619. doi:

10.1016/j.healthplace.2021.102619.

31. Mitra R, Moore SA, Gillespie M, et al. Healthy movement behaviours in children and youth during the COVID-19 pandemic: Exploring the role of the neighbourhood environment. Health Place 2020;65:102418. doi: 10.1016/j.healthplace.2020.102418.

32. Field T. Social media and mental health in youth during COVID-19: A narrative review. Int J Psychol Res. 2021;4:54. doi.org/10.28933/ijprr-2021-08-2905.
33. Guerrero MD, Vanderloo LM, Rhodes RE, Faulkner G, Moore SA, Tremblay MS. Canadian children's and youth's adherence to the 24-h movement guidelines during the COVID-19 pandemic: A decision tree analysis. J Sport Health Sci 2020;9:313-21. doi: 10.1016/j.jshs.2020.06.005.

34.Zheng Y, Wang $W$, Zhong $Y$, et al. A peer-to-peer livestreaming intervention for children during COVID-19 homeschooling to promote physical activity and reduce anxiety and eye strain: Cluster randomized controlled trial. J Med Internet Res 2021;30;23:e24316. doi: 10.2196/24316.

35. O'Kane SM, Lahart IM, Gallagher AM, et al. Changes in physical activity, sleep, mental health, and social media use during COVID-19 lockdown among adolescent girls: A mixed-methods study. J Phys Act Health 2021;18:677-85. doi: 10.1123/jpah.2020-0649.

36. Field T. Massage therapy research: A narrative review. Int J Psychol Res Rev. 2021;4:45. doi: 10.28933/ijprr2020-12-0805. 